\title{
Structural and Magnetic Properties of Co Thin Films on $\mathrm{Au}(111)$ Substrates
}

\author{
A. Walczak*, T. Ślusarski, A. Lehmann-Szweykowska and G. Kamieniarz \\ Faculty of Physics, Adam Mickiewicz University, Umultowska 85, 61-614 Poznań, Poland
}

\author{
(Received May 13, 2011)
}

\begin{abstract}
The structural and magnetic properties of thin Co films grown on $\mathrm{Au}(111)$ substrates are investigated using $a b$ initio local-spin-density calculations in the generalized gradient approximation. It is shown that a large lattice intermismatch between $\mathrm{Co}$ and $\mathrm{Au}(111)$ leads to a strong contraction of the interlayer distances within the Co layer, which adopts either the hexagonal close-packed ( $\alpha$-phase) or the face-centered cubic ( $\beta$-phase) structure of bulk Co. Magnetic moments in the Co layer are increased beyond their value in bulk Co, the enhancement being strongest at the free surface and at the $\mathrm{Co} / \mathrm{Au}$ interface, and more modest in the central part of the adlayer. Moderating influence of capping Ag layers on the structure and the magnetic properties of the films has also been considered. It is shown that Ag capping reduces the magnetic moments in comparison to uncovered Co/Au systems.
\end{abstract}

PACS: 75.70.-i, 68.35.-p

\section{Introduction}

Magnetic thin films in a monolayer regime have revealed a variety of interesting physical phenomena such as perpendicular magnetic anisotropy (PMA) [1-5], enhanced magnetic moments at the surface and interface, oscillatory behaviour of the interlayer magnetic coupling [6], and giant magnetoresistance [7]. The magnetic properties have been considered to play a prominent role in developing future magnetic data-storage media. Magnetic $\mathrm{Co}-\mathrm{Au}$ system is of particular interest, since in addition to its role in the study of PMA in thin magnetic films, it is also a promising candidate for magneto-optical storage technology. Magnetic anisotropy study shows that PMA is strongly influenced by the expansion of the in-plane lattice of Co. It suggests that the connection between the structure and electronic states has an influence on the magnetic properties of Co films at the interface.

Since the magnetic properties of the systems depend on details of geometric structures, many studies have been performed, using various experimental techniques. In $\mathrm{Co} / \mathrm{Au}(111)$ system, a large lattice mismatch $(\approx 14 \%)$ between $\mathrm{Co}$ and $\mathrm{Au}$ causes the characteristic growth mode of Co films. The features of the growth mode and the structure of Co were observed in detail using surface X-ray absorption spectroscopy (EXAFS) [8, 9], scanning tunneling microscopy (STM) $[10,11]$ in combination with the molecular-beam epitaxy (MBE) [9, 12], X-ray diffraction study [13], core level photoemission spectroscopy [14], low-energy electron diffraction (LEED), the Auger electron spectroscopy (AES) [1, 10, 15] and ion scattering experiments [16]. Morphological information is needed for understanding the characteristic ferromagnetism of Co film.

EXAFS experiment and X-ray diffraction measurements indicate that $\mathrm{Co} / \mathrm{Au}(111)$ layers have a quasiper-

* corresponding author; e-mail: annaw@amu.edu.pl fect hcp structure for films thicker than $4 \mathrm{ML}[8,14]$. In this regime the huge lattice strain can be released by forming misfit dislocation. The dislocation regions appear as $0.15 \AA$ high ridges separating the fcc from hcp regions. They are shown in STM image [11]. The structure of ultrathin films, formed during very early stages of the growth, is of particular interest since there appear interface alloying, surface and interfacial stress [9, 13]. The interface $\mathrm{Co} / \mathrm{Au}$ can be also well described by EXAFS technique.

Recent studies of the closely related $\mathrm{Co} / \mathrm{Pt}(111)$ system [17] show that films, grown at low temperature $(120 \mathrm{~K})$, adopt hcp structure with a small concentration of stacking faults, whereas films of the same thickness, grown at room temperature, contain a high number of stacking faults and have predominantly fcc structure. This result seems to be consistent with a similar empirical observation, confirmed by the $a b$ initio calculations, concerning bulk Co [18].

In this paper, we show enhancement of magnetic moment at the free $\mathrm{Co}$ surface and at the $\mathrm{Co} / \mathrm{Au}(111)$ interface. The interfacial magnetic moments have been remarkably enhanced as compared with the bulk values. We have found that the surface contraction in $(x) \mathrm{Co} /$ $\mathrm{Au}(111)$ films is the same as for bulk hcp Co with in-plane lattice constant of Au. Here, we present the structure and magnetic properties of $\mathrm{Co} / \mathrm{Au}(111)$ and $\mathrm{Ag} / \mathrm{Co} / \mathrm{Au}(111)$ sandwich structures using $a b$ initio spin-density (DFT) calculations.

In Sect. 2 computational details are described. In Sect. 3 we study energy and magnetism of uniaxially distorted hcp and fcc Co. Section 4 contains results of uncapped $(x) \mathrm{Co} / \mathrm{Au}(111)$ film structure. In Sect. 5 we explore the effect of the capping $\mathrm{Ag}$ layer and we report results for $\mathrm{Ag} / \mathrm{Co} / \mathrm{Au}(111)$ sandwiches with varying thickness of the Ag and Co film, respectively. In Sect. 6 we study growth mode for capped and uncapped Co films due to the islands formation. Also, alloying preference in $(2 \times 1)$ surface cell is presented. Finally, in Sect. 7 our main results are briefly summarised. 


\section{The method}

The properties of $\mathrm{Co} / \mathrm{Au}(111)$ and $\mathrm{Ag} / \mathrm{Co} / \mathrm{Au}(111)$ systems have been computed using first principle spin-density functional theory in two mutually complementing codes. First, we use the exchange-correlation functional of Perdew and Zunger [19], adding generalised gradient correction (GGC) proposed by Perdew and Wang (PW) [20]. The Kohn-Sham equations are solved using the projector-augmented wave technique [21] as implemented in the VIENNA ab initio simulation program [22-24]. Structural relaxations are performed via quasi-Newton algorithm, using the exact Hellmann-Feynman forces acting on the ions.

In the second version, electronic and magnetic properties of $\mathrm{Co} / \mathrm{Au}(111)$ and $\mathrm{Ag} / \mathrm{Co} / \mathrm{Au}(111)$ systems are studied using SIESTA package ([25] and refs. therein). SIESTA uses linear combinations of numerical localised atomic-orbital basis sets for description of valence electrons and norm-conserving non-local pseudopotentials for the atomic core. Exchange and correlation effects are accounted for by the generalised gradient local approximation (GGA) in the Perdew-Burke-Erzenhof (PBE) version. We carry out the SIESTA calculations using the same geometrical structure as that in VIENNA. We use DZP (double- $\zeta$ polarised) basis set and mesh-off greater than 200 Ry. For density matrix tolerance parameter set to $10^{-6}$, the total energy varies of around few tenth of $\mathrm{meV}$. We use standard pseudopotentials dedicated for SIESTA with the Troullier-Martins scheme. Also, the scalar-relativistic effects and non-linear core corrections are taken into account for $\mathrm{Au}, \mathrm{Co}$, and $\mathrm{Ag}$ atoms. Apart from aiming at computing some new characteristics observed experimentally, an additional motivation for this work was to compare whether calculations based on the SIESTA code give reliable results consistent with those obtained by the VIENNA code.

\section{TABLE I}

Lattice constant a $[\AA]$, surface energy $\sigma$ [eV/atom], and surface relaxation $\Delta d_{12}[\%]$ for $\mathrm{Co}, \mathrm{Au}$, and $\mathrm{Ag}(x) \mathrm{Co} / \mathrm{Au}(111)$ system.

\begin{tabular}{c|c|c|c|c|c}
\hline \hline Metal & & $a$ & $\sigma$ & $\Delta d_{12}$ & Ref. \\
\hline $\mathrm{Co}(\mathrm{hcp})$ & th. & 2.50 & 0.64 & -0.7 & \\
& exp. & 2.51 & 0.58 & -0.7 & {$[29]$} \\
$\mathrm{Au}($ fcc) & th. & 4.18 & 0.33 & 0.3 & \\
& exp. & 4.07 & 0.27 & 1.5 & {$[9]$} \\
$\mathrm{Ag}($ fcc $)$ & th. & 2.89 & 0.55 & -1.4 & {$[28]$} \\
& exp. & 2.04 & & & {$[35]$}
\end{tabular}

In our model a slab of six Au layers represents the substrate, which is covered by a varying number of $\mathrm{Co}$ layers, plus eventual Ag capping layers. Brillouin-zone sampling has been performed using the Monkhorst-Pack grids [26] and level broadening based on the MethfesselPaxton functions [27]. The calculations for complete adlayers and for surface alloying have been performed using
$(1 \times 1)$ surface cells and $(2 \times 1)$ cells, respectively. For the $(1 \times 1)$ geometry grids up to $(15 \times 15 \times 2)$ corresponding to $54 k$ points in the irreducible wedge has been used. The calculations for surface alloys in a $(2 \times 1)$ geometry were performed using $(10 \times 6 \times 1)$ grids $(24$ irreducible $k$ points). The vacuum gap separating the periodically repeated slab was chosen to be $4 \mathrm{ML}$. The details apply to both codes.

All calculations have been carried out using the equilibrium lattice constant of $\mathrm{Au}$ calculated with the GGC $\left(a_{\mathrm{Au}}=4.18 \AA, \tilde{a}_{\mathrm{Au}}=4.07 \AA\right)$ [10] for the frozen part of the Au substrate. For the three metals, considered in our study, we have also calculated the surface energies and the relaxation of a clean (111) surface. The results are gathered in Table I, together with the available experimental results.

The surface energies have been calculated using the well-known relation $\sigma=\left(E_{\text {slab }}-n E_{\text {bulk }}\right) / 2$, where $E_{\text {slab }}$ stands for the total energy of the slab containing $n$ atoms per cell and $E_{\text {bulk }}$ for the cohesive energy per atom of the bulk metal. We note that while for Co and $\mathrm{Ag}$, the gradient corrected functionals predict lattice constants in good agreement with the experiment, for heavy metals, however, such as Au, the GGC tends to correct the over-binding tendency of the local density approximation. For the close-packed surfaces, like those considered here, LEED experiments $[28,30,31]$ show only very weak surface relaxations, in good agreement with our calculations.

\section{Magnetism of uniaxially distorted hep Co}

We start by considering the total energy of bulk hcp Co constrained in such a way that the basal plane of its hcp structure matches geometry of the (111) surface of fcc $\mathrm{Au}$ with a calculated in-plane nearest-neighbour distance of $d=a_{\mathrm{hcp}}=2.956 \AA$. Contour plots of both the total energy and of the magnetic moment are shown in Fig. 1.

As the hexagonal lattice is gradually expanded in the basal plane, the axial ratio is contracted in order to conserve as far as possible the atomic volume. If the $\mathrm{Co}(0001)$ plane is lattice-matched to $\mathrm{Au}(111)$, the total energy is minimised at an axial ratio of $c / a=0.715$, corresponding to an interlayer contraction of about $30 \%$ and a modest volume expansion of $17.5 \%$. The volume expansion leads also to a modest increase of the magnetic moment. Cuts through the potential-energy and magnetic-moment surfaces at fixed $a_{\mathrm{hcp}}=2.956 \AA$ are presented in Fig. 2.

\section{Uncapped $(x) \mathrm{Co} / \mathrm{Au}(111)$ films}

Metallic cobalt can crystallise in three different crystal structures. Two of the phases $(\alpha$ and $\beta$ ) are based on close packing of atoms although they differ in the stacking sequence of (111)-plane. These phases possess similar energetic stability and, hence, small temperature or pressure variations give rise to changes in the crystal phase. In both crystal phases ferromagnetic structure is the most stable magnetic phase. Although $\alpha$-Co and $\beta$-Co can even coexist in the same sample, the first phase is more 


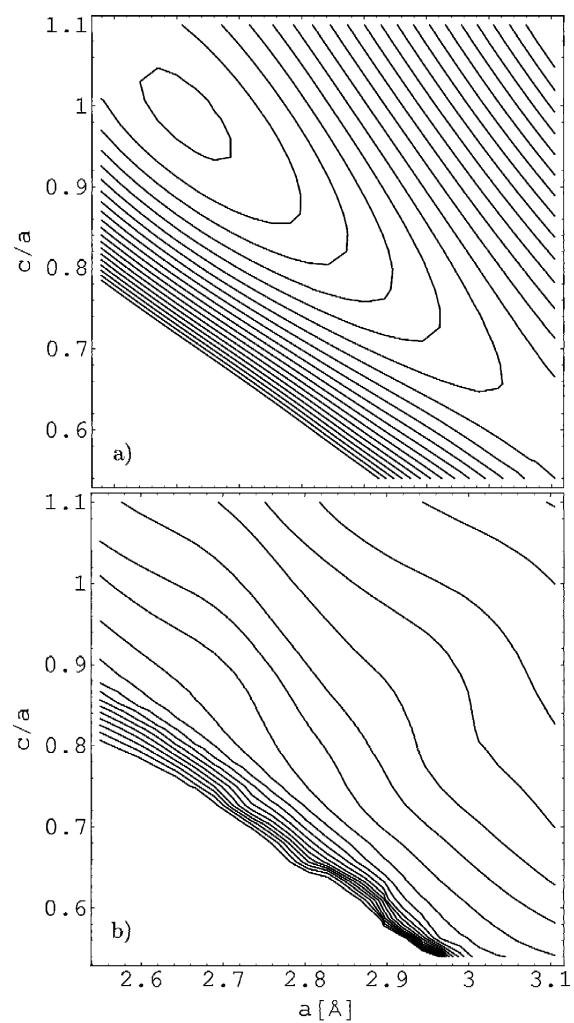

Fig. 1. Contour plots of the total energy (a) and of the magnetic moment (b) of hexagonal $\mathrm{Co}$ as a function of both the lattice constant $a$ (in $\AA$ ) in the hexagonal plane and the axial ratio $c / a$ (given in units of the ideal hcp value). The minimum energy in the hcp ground-state with $c / a=0.72$ is $-6.56 \mathrm{eV} /$ atom. Constant-energy contours are drawn at intervals of $0.1 \mathrm{eV}$. In equilibrium, the calculated magnetic moment is $1.77 \mu_{\mathrm{B}}$ with contour intervals equal to $0.05 \mu_{\mathrm{B}}$.

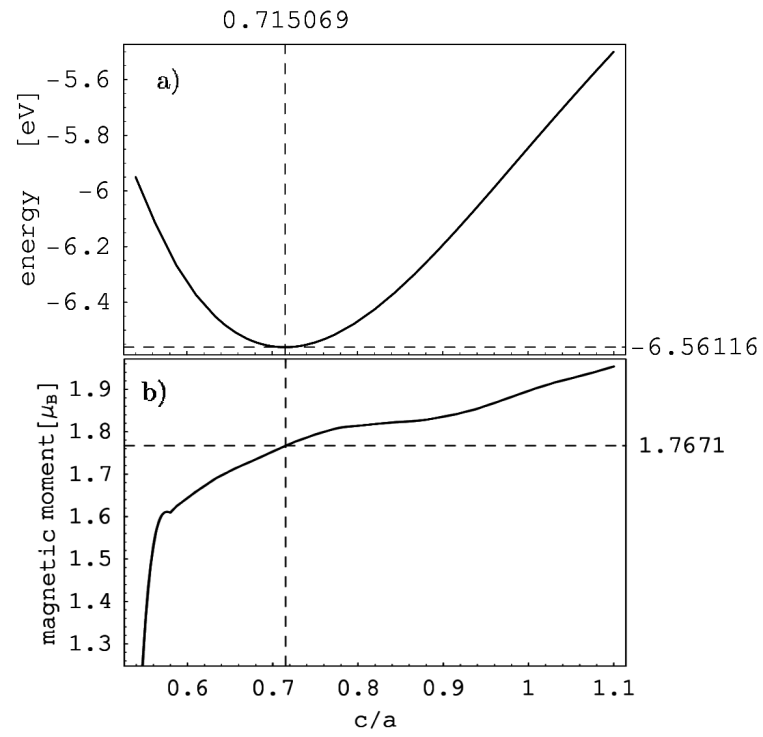

Fig. 2. (a) Energy and (b) magnetic moment vs. $c / a$ in hcp Co with the in-plane lattice parameter constrained to match the (111) surface of $\mathrm{Au}(a=2.956 \AA)$. stable at room temperature and ambient pressure [18]. For Co films grown on $\mathrm{Au}(111)$ the essential question is whether the film will continue the fcc stacking sequence of the substrate, adopt the native hcp sequence of $\alpha$-Co, or form stacking faults representing a compromise between the structural tendencies of $\mathrm{Co}$ and $\mathrm{Au}$. For films with up to six monolayers of Co we have considered a variety of possible configurations. The results are compiled in Table II.

\section{TABLE II}

Total energy $E[\mathrm{eV} /$ cell], energy difference relative to the ground-state configuration $\Delta E[\mathrm{meV} / \mathrm{Co}$ atom], energy of formation (per surface atom) $\sigma_{\mathrm{f}}[\mathrm{eV} /(\mathrm{Co}$ atom) $]$ and average Co moments $\bar{m}_{\mathrm{Co}}\left[\mu_{\mathrm{B}}\right]$ in $\mathrm{Co} / \mathrm{Au}(111)$ films with varying stacking sequences in the Co film.

\begin{tabular}{c|c|c|c|c}
\hline \hline Configuration & $E$ & $\Delta E$ & $\sigma_{\mathrm{f}}$ & $\bar{m}_{\mathrm{Co}}$ \\
\hline $\mathrm{Au}(111)$ & -18.5489 & 0.0 & & \\
\hline \multicolumn{5}{c}{$1 \mathrm{Co} / \mathrm{Au}(111)$} \\
\hline $\mathrm{A} / \mathrm{CBA}$ & -24.1599 & 0.0 & 1.27 & 1.93 \\
$\mathrm{~B} / \mathrm{CBA}$ & -24.1321 & 28.9 & 1.29 & 1.92 \\
\hline \multicolumn{5}{c}{$2 \mathrm{Co} / \mathrm{Au}(111)$} \\
\hline $\mathrm{CA} / \mathrm{CBA}$ & -31.1163 & 0.0 & 0.89 & 1.95 \\
$\mathrm{CB} / \mathrm{CBA}$ & -31.0965 & 9.9 & 0.89 & 1.94 \\
$\mathrm{BA} / \mathrm{CBA}$ & -31.0947 & 10.8 & 0.89 & 1.96 \\
$\mathrm{AB} / \mathrm{CBA}$ & -31.0734 & 21.4 & 0.91 & 1.95 \\
\hline \multicolumn{5}{c}{$3 \mathrm{Co} / \mathrm{Au}(111)$} \\
\hline ACA/CBA & -37.6034 & 0.0 & 0.95 & 1.89 \\
$\mathrm{ABA} / \mathrm{CBA}$ & -37.5865 & 5.6 & 0.97 & 1.89 \\
$\mathrm{CBA} / \mathrm{CBA}$ & -37.5733 & 10.0 & 0.99 & 1.90 \\
\hline \multicolumn{5}{c}{$4 \mathrm{Co} / \mathrm{Au}(111)$} \\
\hline $\mathrm{CACA} / \mathrm{CBA}$ & -44.1955 & 0.0 & 0.91 & 1.90 \\
$\mathrm{BABA} / \mathrm{CBA}$ & -44.1729 & 5.7 & 0.95 & 1.89 \\
$\mathrm{ACBA} / \mathrm{CBA}$ & -44.1080 & 21.9 & 1.01 & 1.93 \\
\hline \multicolumn{5}{c}{$5 \mathrm{Co} / \mathrm{Au}(111)$} \\
\hline ACACA/CBA & -50.7137 & 0.0 & 0.97 & 1.88 \\
ABABA/CBA & -50.6833 & 6.1 & 0.99 & 1.87 \\
$\mathrm{BACBA} / \mathrm{CBA}$ & -50.6312 & 16.5 & 1.05 & 1.92 \\
\hline
\end{tabular}

For a Co monolayer (ML), Co positions continue the fcc-like stacking sequence of the substrate (i.e. A/CBACBA), the energy-penalty for a stacking fault (B/CBACBA) at the interface is $28 \mathrm{meV} /(\mathrm{Co}$ atom). For a 2 ML Co-film, however, the second Co-layer begins an hcp-like sequence in the adlayer, and even on the side of the film interface this sequence is not disturbed, i.e. CA/CBACBA remains the stable sequence. This hcp-Co/fcc-Au configuration (CACACA/ CBACBA) remains the ground-state up to the thickest $6 \mathrm{ML} \mathrm{Co} / \mathrm{Au}(111)$ films covered in this study. Experiment also shows clearly a hexagonal stacking for films thicker than $4 \mathrm{ML}$, as evidenced by the polarisation dependence of the XANES and EXAFS spectra, taken at the Co $K$-edge [14]. The structural energy differences pe- 
nalising other stacking sequences decrease with increasing film thickness, for the $6 \mathrm{ML}$ film the energy difference between the hcp/fcc (CACACA/CBACBA) and the fcc/fcc (CBACBA/CBACBA) configurations is only $15 \mathrm{meV} /(\mathrm{Co}$ atom $)$. The energies for mixed hcp/fcc sequences in the Co-films range between these two extremes - i.e. stacking faults can be easily formed at elevated growth temperatures. It seems that one should expect just the opposite relation between the penalty energy for the faulty stacking and the film thickness. The result, however, can be interpreted in terms of $\alpha$-Co and $\beta$-Co possible coexistence as has been already reported in bulk Co [18], where also low energy is required for stacking fault formation. Moreover, the actual conclusion opens a new perspective with respect to possible origins of anomalous magnetic anisotropy in such systems (see: for instance, [4]).

The energy of formation $\sigma_{\mathrm{f}}$ (per surface atom) is defined as [32]:

$$
\begin{array}{r}
\sigma_{\mathrm{f}}= \\
{\left[E_{\mathrm{slab}}(\mathrm{Co} / \mathrm{Au})-N_{\mathrm{Co}} E_{\mathrm{Co}}^{\mathrm{bulk}}-N_{\mathrm{Au}} E_{\mathrm{Au}}^{\mathrm{bulk}}\right] / A-\sigma_{\mathrm{Au}},}
\end{array}
$$

where $A$ is the number of atoms per surface cell, $N_{\mathrm{Au}}$ and $N_{\text {Co }}$ are the numbers of $\mathrm{Co}$ and $\mathrm{Au}$ atoms in the supercell, $E_{\mathrm{Co}}^{\mathrm{bulk}}$ and $E_{\mathrm{Au}}^{\mathrm{bulk}}$ are the energies (per atom) of $\alpha$-Co and $\mathrm{Au}$ in their respective crystal structures at the theoretical equilibrium lattice constants. The last term corrects for the fact that asymmetric slabs have been used and the lower surface in the supercells is an unrelaxed (bulk-terminated) Au surface. For a pure Au slab $\sigma_{\mathrm{f}}$ is hence just the $\mathrm{Au}(111)$ surface energy, for an overlayer the energy of formation is given essentially by the surface energy of $\alpha$-Co plus the $\mathrm{Co} / \mathrm{Au}$ interface energy.

Our results for the relaxed interlayer distances and the layer-resolved magnetic moments of the energetically most favourable configurations are compiled in Table III. As expected from our results on uniaxially distorted hcp $\mathrm{Co}$, the interlayer distances in the $\mathrm{Co} / \mathrm{Au}(111)$ films are contracted on average by about $30 \%$ compared to the substrate. The interlayer contraction is greater at the free surface (about 34\%) and smaller (around 28\%) in the central part of the film. At the interface, the distance between $\mathrm{Co}$ and $\mathrm{Au}$ is contracted only by about $12 \%$, reflecting the weak coupling between the substrate and the adlayer.

Magnetic moments in the Co film are considerably enhanced above their value in bulk Co $\left(m_{\mathrm{Co}}=1.62 \mu_{\mathrm{B}}\right)$, to almost $2 \mu_{\mathrm{B}}$. The enhancement is strongest at the free surface, where the effect of a reduced coordination number (and hence a narrowing $d$-band width) adds to the effect of the enhanced volume due to the size mismatch. At the $\mathrm{Co} / \mathrm{Au}$ interface, as well, we find a moment $\left(\approx 1.9 \mu_{\mathrm{B}}\right)$ larger than in the interior of the films $\left(\approx 1.8 \mu_{\mathrm{B}}\right)$. Only minimal magnetic moments $(\approx$ $\left.0.01 \mu_{\mathrm{B}}\right)$ are induced in the top layer of the Au substrate - this remains in contrast to the strong magnetic doping effects found for Co layers on top of transition-metal sub-
TABLE III

The changes in interlayer distances $\Delta d_{i, j}$ (in \%) and the local magnetic moments $m_{i}\left(\right.$ in $\left.\mu_{\mathrm{B}}\right)$ in hcp and fcc $6 \mathrm{ML} \mathrm{Co/Au(111)} \mathrm{films.}$

\begin{tabular}{c|c|c|c|c}
\hline \hline & $\begin{array}{c}\Delta d_{i, j} \\
\mathrm{hcp}\end{array}$ & $\begin{array}{c}\Delta d_{i, j} \\
\text { fcc }\end{array}$ & $\begin{array}{c}m_{i} \\
\text { hcp }\end{array}$ & $\begin{array}{c}m_{i} \\
\text { fcc }\end{array}$ \\
\hline $\mathrm{Co}(1)$ & -33.7 & -33.9 & 1.96 & 1.95 \\
$\mathrm{Co}(2)$ & -27.8 & -26.1 & 1.86 & 1.92 \\
$\mathrm{Co}(3)$ & -29.2 & -28.1 & 1.81 & 1.88 \\
$\mathrm{Co}(4)$ & -28.1 & -27.5 & 1.82 & 1.90 \\
$\mathrm{Co}(5)$ & -31.4 & -31.4 & 1.86 & 1.92 \\
$\mathrm{Co}(6)$ & -12.3 & -11.7 & 1.87 & 1.85 \\
$\mathrm{Au}(1)$ & 0.9 & 0.8 & 0.01 & -0.01 \\
$\mathrm{Au}(2)$ & 0.7 & 0.4 & - & -
\end{tabular}

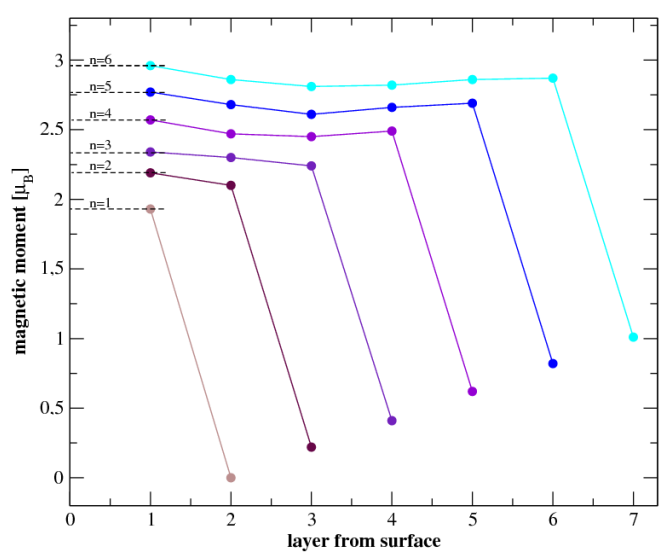

Fig. 3. Magnetic moment profiles for hcp Co on $\mathrm{Au}(111)$, showing the profiles at the surfaces of hcp Co and $\mathrm{fcc} \mathrm{Au}(111)$. Values of the moment are shifted by $0.2(n-1) \mu$ with increasing number of Co monolayers.

strates (see Fig. 3 and e.g. Ref. [33] and further references therein). Hence, the noble-metal substrates prove to be magnetically inert. It is also interesting that the magnetic profiles and the geometric details of the $\mathrm{Co} / \mathrm{Au}(111)$ films are only moderately influenced by the stacking sequence.

As an example, we present in Table III the result for $\alpha$ and $\beta 6 \mathrm{ML}$ Co films. In the hcp films, the interlayer contraction is slightly more pronounced in the central part of the film - this is also reflected in a somewhat more corrugated magnetic profile. In $\mathrm{Co} / \mathrm{Au}(111)$ film intermixing is strongly preferred. Co moments are only slightly reduced by intermixing. We have noticed buckling of surface and subsurface layer, but local volume of Co is conserved (see Table IV).

\section{Silver-capped Co/Au(111) films}

As transition-metal films tend to be subject to strong oxidation, it is customary to protect the films by inert capping layers. We have explored the influence of capping $\mathrm{Ag}$ layers on top of the $\mathrm{Co} / \mathrm{Au}(111)$ films. In the first step we have examined $1 \mathrm{ML} \mathrm{Co} / \mathrm{Au}(111)$ covered by one to six Ag ML's, varying the stacking sequence in the 
TABLE IV

Total energy $E$ [eV/cell], local magnetic Co moments $\mathrm{cm}\left[\mu_{\mathrm{B}}\right]$ and surface relaxation $s r[\%]$ in $(2 \times 1)$ surface cell, $50 / 50$ surface/alloy subsurface.

\begin{tabular}{l|c|c|c}
\hline \hline & $E$ & $\mathrm{~cm}$ & $s r$ \\
\hline unalloyed $2 \times 1$ & -48.2955 & & \\
$\quad$ Co/Au(111) cell & & 1.97 & -16.0 \\
1st layer from surface & & 1.97 & -16.0 \\
2nd layer from surface & & \\
alloyed $2 \times 1$ & -49.1123 & & \\
1Co/Au(111) cell & & \\
1st layer from surface (Co) & & \\
2nd layer from surface (Au) & & & -23.1 \\
3rd layer from surface (Co) & & 1.94 & \\
4th layer from surface (Au) & & &
\end{tabular}

Ag-caps. We have found that the Ag-layers always prefer to adopt an fcc stacking sequence, for a 6 ML Ag-cap the ground-state configuration is ACBACB/A/CBACBA (and a part thereof for thinner capping layers). However, stacking faults in the Ag-cap are penalised only by minimal energy differences of the order of $1 \mathrm{meV}$. It is more interesting to examine the geometric structure and the magnetic properties of the $\mathrm{Ag} / \mathrm{Co} / \mathrm{Au}(111)$ complex. The calculated inter-layer relaxations are compiled in Table V. We find that the $\mathrm{Co} / \mathrm{Au}$ layer distances at the

\section{TABLE V}

The changes in interlayer-distances $d_{i, j}[\%]$ and the local magnetic moments in the $\mathrm{Ag} / 3 \mathrm{Co} / 6 \mathrm{Au} A 1$ and $\mathrm{Ag} / 5 \mathrm{Co} /$ $6 \mathrm{Au} A 2$ configurations.

\begin{tabular}{c|c|c|c|c}
\hline \hline & $\Delta d_{i, j}$ & $\Delta d_{i, j}$ & $m_{i}$ & $m_{i}$ \\
& $A 1$ & $A 2$ & $A 1$ & $A 2$ \\
\hline $\mathrm{Au}(1)$ & - & - & - & - \\
$\mathrm{Au}(2)$ & - & - & - & - \\
$\mathrm{Au}(3)$ & - & - & - & - \\
$\mathrm{Au}(4)$ & - & -0.2 & - & - \\
$\mathrm{Au}(5)$ & 0.5 & 0.7 & - & - \\
$\mathrm{Au}(6)$ & 0.8 & 0.7 & 0.01 & 0.01 \\
$\mathrm{Co}(1)$ & -12.7 & -12.6 & 1.83 & 1.85 \\
$\mathrm{Co}(2)$ & -30.4 & -31.5 & 1.88 & 1.84 \\
$\mathrm{Co}(3)$ & -31.0 & -28.2 & 1.81 & 1.78 \\
$\mathrm{Co}(4)$ & - & -27.9 & - & 1.84 \\
$\mathrm{Co}(5)$ & - & -32.4 & - & 1.83 \\
$\mathrm{Ag}(1)$ & -10.4 & -10.2 & -0.01 & -0.01 \\
$\mathrm{Ag}(2)$ & -0.4 & -0.8 & - & -0.01 \\
$\mathrm{Ag}(3)$ & -1.6 & -1.8 & - & -0.01
\end{tabular}

interface are marginally expanded by about one percent, the $\mathrm{Ag} / \mathrm{Co}$ distance being slightly larger (by about $3 \%$ of the inter-layer distance in bulk $\mathrm{Au}$ ). The inter-layer distances in the capping $\mathrm{Ag}$ film are slightly contracted as expected from the size mismatch between $\mathrm{Ag}$ and $\mathrm{Au}$. Magnetic moments in the covered Co ML are reduced by 0.05 to $0.09 \mu_{\mathrm{B}}$ compared to the clean Co films (Fig. 4).

To examine the influence of a capping layer on the properties of thicker $\mathrm{Co} / \mathrm{Au}(111)$ films, we have exam-

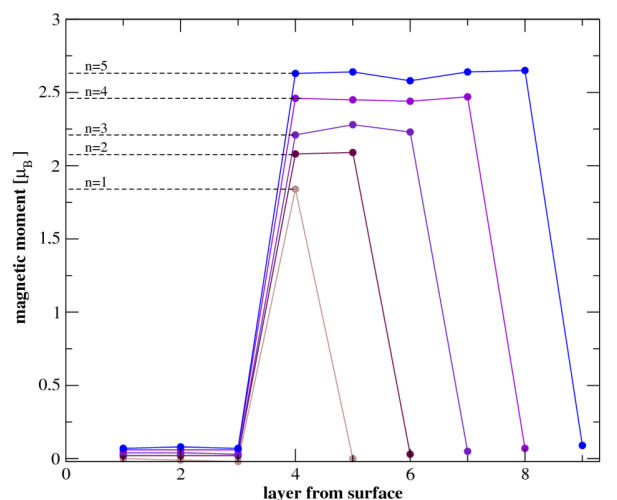

Fig. 4. Magnetic moment profiles for $3 \mathrm{Ag} /(x) \mathrm{Co} /$ $\mathrm{Au}(111)$ structure, showing the profiles at the interfaces. Moment values are shifted by $0.2(n-1) \mu$ with increasing number of Co monolayers.

ined $3 \mathrm{Ag} / x \mathrm{Co} / \mathrm{Au}(111)$ films with $x$ varying between 1 and 6. We have assumed hcp stacking for cobalt, as $\alpha$-Co is the most stable Co phase at lower temperatures [18]. The assumption is also confirmed in glancing angle X-ray diffraction (GXRD) experiments for similar sandwich structures [34]. The stacking sequence has been varied, however, in the capping Ag layer. The stable configurations are found to be $\mathrm{ACB} / \mathrm{A} / \mathrm{CBACBA}$ for $1 \mathrm{ML}$ $\mathrm{Co}, \mathrm{CBA} / \mathrm{CA} / \mathrm{CBACBA}$ for $2 \mathrm{ML} \mathrm{Co}$ and so forth for thicker layers, i.e. the configurations tend towards fcc/ hcp/fcc, with an arrangement at both interfaces that fits both the fcc stacking in the Au substrate and in the Ag cap, and the hcp stacking in the Co inter-layer. Stacking fault energy in the Ag-cap is in most cases of the order of $1 \mathrm{meV}$, and never exceeds $3 \mathrm{meV}$.

Information on the energy of formation as well as the structural and magnetic properties is compiled in Table $\mathrm{V}$. The energy of formation is given essentially by the surface energy of $\mathrm{Ag}$ plus the $\mathrm{Ag} / \mathrm{Co}$ and $\mathrm{Co} / \mathrm{Au}$ interface energies. Silver capping causes an increase of stability in $\mathrm{Co} / \mathrm{Au}(111)$ films. Changes in the film geometry relative to that of the uncovered films are restricted to a more modest contraction of the distance between the Co-layers on the Ag-side of the film (compared to the layers near the free films surface), and to a reduction (by about $0.1 \mu_{\mathrm{B}}$ ) of the Co-moments at the $\mathrm{Co} / \mathrm{Ag}$ interface relative to the free surface.

\section{Island formation}

From various factors, complicating the growth of ultrathin films of $\mathrm{Co}$ on $\mathrm{Au}(111)$, we focus on island formation. If the surface energy of the substrate is substantially lower than that of the metal forming the overlayer, the island formation will be preferred at least in the initial stages of growth. Under the assumption that the islands are sufficiently large so that contributions from the step edges and side facets of the islands can be neglected, the energy of a system consisting of more than one domain (e.g. of regions of the clean Au surface and regions of Co islands of different thickness) is simply given by the 
weighted average of the surface energy of the substrate $\mathrm{Au}$ ) and of the energy of formation of $\mathrm{Co} / \mathrm{Au}$ films of corresponding thickness. For the decomposition of a Co film of $(n+1)$ ML (clean or Ag covered) into $n$ ML thick homogeneous basis part and islands with a step height of $h \mathrm{ML}$, the energy difference can be evaluated according to [32]:

$$
\begin{aligned}
& \Delta E_{\mathrm{isl}}^{h, n}=\left[(h-1) E_{n \mathrm{Co} / \mathrm{Au}}+E_{(h+n) \mathrm{Co} / \mathrm{Au}}\right. \\
& \left.\quad-h E_{(n+1) \mathrm{Co} / \mathrm{Au}}\right] / h,
\end{aligned}
$$

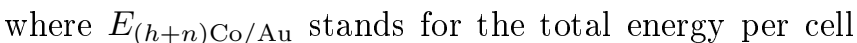
of a $(h+n) \mathrm{Co} / \mathrm{Au}(111)$ slab. Negative values of island formation energy $\Delta E_{\text {isl }}^{h, n}$ mean favourable conditions for the process. Table VI summarises the island-formation energy for up to $4 \mathrm{ML}$ thick Co films on Au surface, both uncapped and capped by a $3 \mathrm{ML} \mathrm{Ag}$ overlayer. As a consequence of the large difference in the surface energies, the preference for the formation of Co-islands on a clean $\mathrm{Au}$ substrate is very pronounced, and it increases with the height of the islands as seen in the first column of Table VII. Once a compact Co-layer has been formed, the tendency for island formation disappears. For thicker Co layers the tendency towards the formation of a rough film seems to be very small.

TABLE VI

Island formation energies (in $\mathrm{eV} /$ surface atom) related to the decomposition of an $(n+1) \mathrm{ML}$ thick film into a $n$ ML homogeneous film covered by $h$ ML high-islands calculated according to Eq. (1) for (1-4) ML Co films on $\mathrm{Au}(111)$ without and with $\mathrm{Ag}$ capping layer.

\begin{tabular}{c|c|c|c|c|c|c}
\hline \hline \multirow{2}{*}{$h$} & \multicolumn{3}{|c|}{ Uncapped film } & \multicolumn{3}{c}{ Capped film } \\
\cline { 2 - 7 } & $n=0$ & $n=1$ & $n=2$ & $n=0$ & $n=1$ & $n=2$ \\
\hline 2 & -0.672666 & 0.234661 & -0.052520 & -0.348674 & 0.119565 & 0.009453 \\
3 & -0.740447 & 0.277868 & -0.012073 & -0.385188 & 0.165721 & -0.032553 \\
4 & -0.800597 & 0.342936 & -0.061838 & -0.398720 & 0.154932 &
\end{tabular}

We detect also an intriguing relation between tendency for the island formation and a number of Co monolayers in the film. Our calculations show that the films with an even number of monolayers should not produce islands in the course of their growth [32]. The results are true both for hcp and fcc Co.

A capping Ag layer strongly reduces the island formation energy, from $\Delta E_{\text {isl }}^{2,0}=-0.67 \mathrm{eV} /$ (surface atom) to $\Delta E_{\mathrm{isl}}^{2,0}=-0.35 \mathrm{eV} /($ surface atom $)$.

\section{Conclusions}

We have investigated some of the properties and the growth mode of ultrathin Co films on $\mathrm{Au}(111)$ substrate by using the density-functional theory.

Our conclusions concern three aspects of the subject of the paper. The magnetic properties of the systems are first. Due to a great deal of interest in theoretical predictions of enhanced magnetic moments in ultrathin ferromagnetic films, this work contains exact values of spin contributions to the magnetic moments for the $\mathrm{Co} / \mathrm{Au}$ system. We have noticed that the Ag overlayer reduces these values. It has been also shown that the lattice mismatch at the $\mathrm{Co}-\mathrm{Au}$ interface induces a hexagonal distortion of the Co films by about $34 \%$. The hexagonal distortion, superposed at the free surface by an inward relaxation of the Co top-layer, is slightly larger than in bulk Co, and enhances the magnetic moment.

The next conclusions are about the geometry of the films and the mode of the growth. We have proved that ACAC... stacking for Co is most stable, and the bulk hcp Co volume is conserved in $(x) \mathrm{Co} / \mathrm{Au}(111)$ thin films. The stacking sequence for the $\mathrm{Ag}$ overlayer has been found to be fcc. The differences in the surface energies of $\mathrm{Au}$ and $\mathrm{Co}$ was the motive to study the growth mode, and we have found roughening in the form of the island formation energetically preferred both for capped and uncapped hcp Co thin films. An interesting aspect is much stronger preference for films with an even number of monolayers [31]. The effect, however, is obtained for the width of terraces much larger than the interatomic spacings. We have also shown that the surface alloying in the $\mathrm{Co} / \mathrm{Au}(111)$ system is strongly preferred.

Moreover, we have also tested VIENNA and SIESTA packages. It has turned out that they provide numerical results, which are in excellent mutual agreement.

For the end we have to add a few remarks about the empirical data. An experimental detailed study of the growth and structure of ultrathin magnetic $\mathrm{Co}-\mathrm{Au}$ films has been carried out by Marsot and others [8] in order to understand their specific magnetic properties from the structural ones. They have also studied the second interface resulting from the $\mathrm{Au} / \mathrm{Co} / \mathrm{Au}$ sandwich in order to investigate the effect of an $\mathrm{Au}$ overlayer in magnetic multilayers. A structural study of the local order in Co thin films by surface EXAFS as well as the glancing angle X-ray diffraction shows that the $\mathrm{Co} / \mathrm{Au}(111)$ has the hexagonal close packed structure and the Au overlayer does not change that. The analysis of the Au $4 f_{7 / 2}$ photoemission line for different Co coverages provides evi- 
TABLE VII

Energies $E[\mathrm{eV} /$ cell $]$, energy difference relative to the ground-state configuration $\Delta E[\mathrm{meV} / \mathrm{Co}$ atom], and average Co moments $\bar{m}_{\mathrm{Co}}\left[\mu_{\mathrm{B}}\right]$ in $(x) \mathrm{Ag} / 1 \mathrm{Co} /$ $\mathrm{Au}(111)$ system.

\begin{tabular}{|c|c|c|c|}
\hline Configuration & $E$ & $\Delta E$ & $\bar{m}_{\mathrm{Co}}$ \\
\hline \multicolumn{4}{|c|}{$1 \mathrm{Ag} / 1 \mathrm{Co} / 6 \mathrm{Au}(111)$} \\
\hline $\mathrm{B} / \mathrm{A} / \mathrm{CBA}$ & -27.3462 & 0.0 & 1.86 \\
\hline $\mathrm{C} / \mathrm{A} / \mathrm{CBA}$ & -27.3392 & 0.7 & 1.86 \\
\hline \multicolumn{4}{|c|}{$2 \mathrm{Ag} / 1 \mathrm{Co} / 6 \mathrm{Au}(111)$} \\
\hline $\mathrm{CB} / \mathrm{A} / \mathrm{CBA}$ & -30.1130 & 0.0 & 1.84 \\
\hline $\mathrm{BC} / \mathrm{A} / \mathrm{CBA}$ & -30.1057 & 0.7 & 1.86 \\
\hline \multicolumn{4}{|c|}{$3 \mathrm{Ag} / 1 \mathrm{Co} / 6 \mathrm{Au}(111)$} \\
\hline $\mathrm{ACB} / \mathrm{A} / \mathrm{CBA}$ & -32.8371 & 0.0 & 1.84 \\
\hline $\mathrm{ABC} / \mathrm{A} / \mathrm{CBA}$ & -32.8294 & 0.8 & 1.85 \\
\hline \multicolumn{4}{|c|}{$4 \mathrm{Ag} / 1 \mathrm{Co} / 6 \mathrm{Au}(111)$} \\
\hline $\mathrm{ACB} / \mathrm{A} / \mathrm{CBA}$ & -35.5240 & 0.0 & 1.86 \\
\hline $\mathrm{ABC} / \mathrm{A} / \mathrm{CBA}$ & -35.5099 & 1.4 & 1.88 \\
\hline \multicolumn{4}{|c|}{$5 \mathrm{Ag} / 1 \mathrm{Co} / 6 \mathrm{Au}(111)$} \\
\hline $\mathrm{ACB} / \mathrm{A} / \mathrm{CBA}$ & -38.3222 & 0.0 & 1.86 \\
\hline $\mathrm{ABC} / \mathrm{A} / \mathrm{CBA}$ & -38.3137 & 0.9 & 1.87 \\
\hline \multicolumn{4}{|c|}{$6 \mathrm{Ag} / 1 \mathrm{Co} / 6 \mathrm{Au}(111)$} \\
\hline $\mathrm{ACB} / \mathrm{A} / \mathrm{CBA}$ & -40.9316 & 0.8 & 1.90 \\
\hline
\end{tabular}

dence of an island growth mode with the presence of a slight interdiffussion process at the interface.

\section{Acknowledgments}

The financial support was provided in part by Polish Ministry of Science and High Education (Grant No N N202 480039 ).

\section{References}

[1] R. Allenspach, M. Stampanoni, A. Bischof, Phys. Rev. Lett. 65, 3344 (1990).

[2] J. Pommier, P. Meyer, G. Pénissard, J. Ferré, P. Bruno, D. Renard, Phys. Rev. Lett. 65, 2054 (1990).

[3] C. Chappert, P. Bruno, J. Appl. Phys. 64, 5736 (1988).

[4] M. Kisielewski, A. Maziewski, M. Tekielak, Phys. Rev. Lett. 89, 087203 (2002).

[5] J. Dubowik, F. Stobiecki, I. Gościańska, K. Röll, J. Magn. Magn. Mater. 272, e923 (2004).

[6] W.B. Zeper, F.J.A.M. Greindamus, P.F. Garcia, C.R. Fincher, J. Appl. Phys. 65, 4971 (1989).

[7] C. Dupas, P. Beauvillain, C. Chappert, J.P. Renard, F. Trigui, P. Veillet, E. Vélu, D. Renaud, J. Appl. Phys. 67, 5680 (1990).

[8] N. Marsot, R. Belkhou, H. Magnan, P. Le Fèvre, C. Guillot, D. Chandesris, Phys. Rev. B 59, 3135 (1999).

[9] L. Cagnon, T. Devolder, R. Cortes, A. Morrone, J.E. Schmidt, C. Chappert, P. Allongue, Phys. Rev. B 63, 104419 (2001)
[10] Ch. Tölkes, R. David, G. Comsa, P. Zeppenfeld, J. Electron Spetrosc. Relat. Phenom. 105, 37 (1999).

[11] B. Voigtländer, G. Meyer, N.M. Amer, Phys. Rev. B 44, 10354 (1991).

[12] T. Koide, H. Miyauchi, J. Okamoto, T. Shidara A. Fujimori, H. Fukutani, K. Amemiya, H. Takeshita, S. Yuasa, T. Katayama, Y. Suzuki, Phys. Rev. Lett. 87, 257201 (2001).

[13] R.J. Nichols, T. Nouar, C.A. Lucas, W. Haiss, W.A. Hofer, Surf. Sci. 513, 263 (2002).

[14] R. Belkhou, N. Marsot, H. Magnan, P. Le Fèvre, N.T. Barrett, C. Guillot, D. Chandesris, J. Electron Spectrosc. Relat. Phenom. 101-103, 251 (1999).

[15] M. Speckmann, H.P. Oepen, H. Ibach, Phys. Rev. Lett. 75, 2035 (1995).

[16] Ch. Tölkes, P. Zeppenfeld, M.A. Krzyżowski, R. David, G. Comsa, Phys. Rev. B 55, 13932 (1997).

[17] C. Quiros, S.M. Valdivares, O. Robach, S. Ferrer, J. Phys., Condens. Matter 17, 5551 (2005).

[18] V.A. de la Peña O'Shea, I. de P.R. Moreira, A. Roldan, F. Illas, J. Chem. Phys. 133, 024701 (2010).

[19] J.P. Perdew, A. Zunger, Phys. Rev. B 23, 5048 (1981).

[20] J. Perdew, J.A. Chevary, S.H. Vosko, K.A. Jackson M.R. Pedersen, D.J. Singh, C. Fiolhais, Phys. Rev. B 46, 6671 (1992).

[21] P. Blöchl, Phys. Rev. B 50, 953 (1994).

[22] G. Kresse, J. Hafner, Phys. Rev. B 48, 115 (1993).

[23] G. Kresse, J. Furthmüller, Phys. Rev. B 54, 169 (1996); Comput. Mater. Sci. 6, 15 (1996).

[24] G. Kresse, D. Joubert, Phys. Rev. B 59, 1758 (1999).

[25] T. Ślusarski, T. Kostyrko, Surf. Sci. 603, 1150 (2009).

[26] H.J. Monkhorst, J.D. Pack, Phys. Rev. B 13, 5188 (1976).

[27] M. Methfessel, A. Paxton, Phys. Rev. B 40, 3616 (1989).

[28] M. Methfessel, D. Hennig, M. Scheffler, Phys. Rev. B 46, 12326 (1992).

[29] S. Lindgren, L. Wallden, J. Rundgren, P. Westrin, Phys. Rev. B 29, 576 (1984).

[30] J.L. Quinn, Ys. Tian, D. Li, H. Jona, F. Marcus, Phys. Rev. B 42, 11348 (1990).

[31] W. Oed, B. Dotsch, L. Hammer, K. Heinz, K. Muller, Surf. Sci. 207, 55 (1988).

[32] D. Spišák, J. Hafner, Phys. Rev. B 64, 205422 (2001).

[33] S. Dennler, J. Hafner, M. Marsman, J. Morillo, Phys. Rev. B 71, 094433 (2005).

[34] R. Belkhou, N. Marsot, H. Magnan, P. Le Fèvre, N. Barrett, C. Guillot, D. Chandesris, J. Electron Spectrosc. Relat. Phenom. 101-103, 251 (1999b).

[35] G.Y. Guo, H. Ebert, W.M. Temmerman, P.J. Durham, Phys. Rev. B 50, 3861 (1994). 